\title{
LA FORMACIÓN PROFESIONAL EN EL ENTORNO DE LA UNIÓN EUROPEA
}

\section{Bonifacio Pedraza}

Licenciado en Ciencias de la Educación por la Universidad Complutense de Madrid

\section{LA CONSTRUCCIÓN DE LA UNIÓN EUROPEA}

Si se intenta resumir brevemente que ha ocurrido en el entorno europeo desde su constitución hasta nuestros días en el ámbito concreto de la formación profesional, se puede llegar a la conclusión de que se ha percibido un cambio de planteamiento entre los tratados constitutivos de la Comunidad Europea, que establecían propuestas para elaborar una política común de formación profesional, con una base jurídica fundamentada en el artículo 128 del Tratado de Roma, artículo que dio pie a la elaboración de los principios generales de 1963 para una política comunitaria de formación profesional, y los tratados posteriores, en concreto en el Tratado de Maastricht, en el que pierden vigencia estos principios de 1963, y en el Tratado de Amsterdam, en el que se define que es competencia de los Estados miembros establecer sus respectivas políticas de formación profesional, y de la Unión Europea facilitar y promover las líneas comunes de actuación, a través de instrumentos que faciliten el intercambio y la definición de políticas que promuevan este acercamiento, con el objeto de facilitar la movilidad de trabajadores y la transparencia de cualificaciones ${ }^{1}$.

Para aclarar cómo ha sido todo este proceso y en qué situación nos encontramos en la actualidad será necesario examinar, por una parte qué es la Unión y cómo se ha configurado y, por otra, cómo se han ido conformando las diferentes políticas educativas y de formación profesional, destacando su vinculación con las políticas de empleo, aspectos que se destacan en los apartados siguientes.

\subsection{Algunas consideraciones previas sobre la Unión Europea}

El proyecto de comunidad europea tuvo su origen en un fuerte contenido de carácter económico. La Unión Europea (UE) es el resultado de un proceso de cooperación e integración que se inició en 1951 entre seis países (Bélgica, Alemania, Francia, Italia, Luxemburgo y los Países Bajos). Tras casi cincuenta años y cuatro tandas de adhesión (1973: Dinamarca, Irlanda y Reino Unido; 1981: Grecia; 1986: España y Portugal; 1995: Austria, Finlandia y Suecia), la UE cuenta hoy con quince Estados miembros y se prepara para su quinta ampliación, esta vez hacia la Europa oriental y del sur. La UE ha asumido como misión organizar de forma coherente y solidaria las relaciones entre los Estados miembros y sus ciudadanos, al igual que los siguientes objetivos esenciales:

- El impulso del progreso económico y social, que se ha concretado en la realización del mercado interior desde 1992 y el lanzamiento de la moneda única en 1999.

\footnotetext{
${ }^{1}$ La Unión Europea elabora reglamentos, que se aplican directamente en cada uno de los Estados miembros sin necesidad de que se aprueben previamente en sus parlamentos, y directivas, que obligan a los Estados miembros a dictar normas o modificar sus leyes en consonancia con lo dispuesto en ellas. La finalidad básica del Derecho comunitario laboral se refiere a la libre circulación de los trabajadores dentro de la Unión Europea y a la equiparación de toda la normativa laboral en el territorio.
} 
- La afirmación de la identidad europea en la escena internacional.

- La implantación de una ciudadanía europea, eliminando barreras y fomentado la movilidad.

- El desarrollo de un espacio de libertad, seguridad y justicia, vinculado a la libre circulación de personas.

- El mantenimiento y desarrollo del acervo comunitario, concretado en los textos jurídicos y los tratados fundacionales.

Si hacemos referencia a la historia de esta unión se podrían destacar los siguientes acontecimientos:

- Como ya se ha comentado, esta Unión tiene su origen en la firma de los tres Tratados fundacionales para la construcción europea, que son los constitutivos de París en 1951 y de Roma en 1957 que instituyeron la Comunidad Europea del Carbón y del Acero (CECA), la Comunidad Económica Europea (CEE) y la Comunidad Europea de la Energía (CEEA o EURATOM).

- Estos tratados fundacionales han sido adaptados y modificados en diversas ocasiones con motivo de la adhesión de nuevos estados miembros. Los Tratados de Adhesión de Dinamarca, Irlanda y Reino Unido (1972), de Grecia (1981) y de España y Portugal (1985) que ampliaron a doce el número de países miembros de la CE y los tratados de adhesión definitiva de Austria, Finlandia y Suecia el 1 de enero de 1995, aumentándose el número de países miembro a quince.

- El Acta Unica Europea (AUE), firmada en Luxemburgo y en La Haya en julio de 1987, en la que se fija una nueva meta para el 31 de diciembre de 1992; la realización de un verdadero mercado interior. Para lograrlo se amplían las competencias de la comunidad a políticas de medioambiente, de investigación y desarrollo tecnológico, e incorpora el principio de la cohesión económica y social, materializado en los fondos estructurales (FEDER, FONDO SOCIAL EUROPEO, FEOGA)

- El Tratado de la Unión Europea, firmado en Maastricht en $1992^{2}$.

- El tratado de Amsterdam de $1999^{3}$, en el que se habla de la Europa del conocimiento y se fijan cuatro grandes objetivos, como son el hacer del empleo y de los derechos de los ciudadanos el eje de la Unión, suprimir los últimos obstáculos a la libre circulación de las personas y reforzar la seguridad, hacer que la voz de Europa se oiga mejor en el mundo y hacer más eficaz la arquitectura institucional de la Unión con miras a la próxima ampliación.

\footnotetext{
${ }^{2}$ Entró en vigor el día 1 de noviembre de 1993. Se destaca el principio de subsidiariedad, como mecanismo de atribución de competencias entre la Unión y los Estados en materias que no sean competencia exclusiva de la Unión. En el Tratado aparece un artículo dedicado al ámbito de formación profesional en el que se destaca la importancia de realizar esfuerzos en la definición y desarrollo de sistemas sólidos que permitan incrementar la formación general y profesional de los ciudadanos europeos.

${ }^{3}$ Entró en vigor el día 1 de mayo de 1999. En el mencionado Tratado, los Estados firmantes se declaran «decididos a promover el desarrollo del nivel más elevado posible de conocimientos para sus pueblos mediante un amplio acceso a la educación y a su continua actualización». En el capítulo dedicado al empleo se establece la necesidad de elaborar un plan nacional sobre empleo anual en cada país miembro.
} 
- El tratado de Niza de 20014. Tras el consejo Europeo de diciembre de 2000, celebrado en Niza, Francia, se firma un nuevo tratado (Tratado de Niza), que modifica el Tratado sobre la Unión Europea y los tratados constitutivos de las comunidades europeas.

En todas estas etapas se percibe siempre la existencia de dos concepciones, la de los «federalistas» que desean avanzar muy rápido hacia la integración y la de los «nacionalistas» que prefieren la simple cooperación intergubernamental a la integración. No obstante la Unión Europea de hoy es fruto del esfuerzo de países que, habiendo vivido las consecuencias de una Europa arrasada por la Segunda Guerra Mundial, quieren crear las bases de un espacio de paz, libertad, estabilidad, crecimiento y bienestar. Se trata de un modelo que persigue la integración europea, dotando a la Comunidad de unos principios y objetivos generales a desarrollar por instituciones propias, capaces de legislar y decidir conforme a un marco jurídico autónomo, y contando con unos recursos presupuestarios independientes.

\subsection{Las instituciones de la Comunidad}

Los objetivos de los tratados mencionados anteriormente y que han dado lugar a la UE actual se aseguran a través de las siguientes instituciones básicas:

- El Consejo de la Unión Europea, órgano que representa a los gobiernos de los Estados miembros, comúnmente conocido como el Consejo de Ministros.

- La Comisión Europea, órgano ejecutivo que ostenta el derecho de iniciativa legislativa.

- El Parlamento Europeo, elegido por sufragio universal directo desde 1979 es el reflejo democrático de la voluntad política de los pueblos de la Unión Europea.

- El Tribunal de Justicia de las Comunidades Europeas, garantiza el respeto y cumplimiento de la legislación comunitaria.

- El Tribunal de Cuentas Europeo, responsable del control de las cuentas, creado en 1975 examina los gastos e ingresos de la Comunidad y es el representante de los contribuyentes, encargado de comprobar que la Unión Europea invierte su dinero siguiendo las normas presupuestarias y para los objetivos a los que está destinado.

Además existe el Consejo Europeo que se crea en 1974 y está compuesto por los Jefes de Estado o de gobierno, acompañados de sus correspondientes Ministros de Asuntos Exteriores, y por el Presidente de la Comisión, al que se une otro miembro de dicha institución. Otros órganos de apoyo son:

- El Comité Económico y Social y el Comité de las Regiones son órganos consultivos que facilitan que se tengan en cuenta las opiniones de los diferentes sectores de la vida económica y social y de las regiones de la Unión Europea.

- El Banco Europeo de Inversiones (BEI), institución financiera de la UE, que concede préstamos a largo plazo y garantías a todos los sectores económicos para favorecer el desarrollo regional

\footnotetext{
${ }^{4}$ Celebrado por el Consejo Europeo de los días 7 a 9 de diciembre de 2000 y firmado el 26 de febrero de 2001, modificará los tratados vigentes. Entrará en vigor una vez que haya sido ratificado por los 15 estados miembros según lo previsto en sus respectivas normativas constitucionales. El proceso de ratificación sigue en marcha y continuará hasta el 2002.
} 
y la realización de inversiones de capital conducentes al equilibrado desarrollo económico y la integración de la Unión.

- El Defensor del Pueblo europeo, que transmite las reclamaciones de los ciudadanos en los casos de mala administración en el plano europeo.

- El Banco Central Europeo, responsable de la política monetaria de la zona euro.

Una vez realizada esta breve presentación del proceso de construcción y estado actual de la UE, se expondrá, en los apartados siguientes, cómo se han ido definiendo, desarrollando y financiando las líneas estratégicas establecidas en los ámbitos de formación y empleo.

\section{ESTRATEGIAS PARA UNA POLÍTICA DE FORMACIÓN PROFESIONAL Y EMPLEO}

\subsection{Los referentes básicos en los Tratados constitutivos}

En el Tratado constitutivo de la CEE, firmado en Roma el 25 de marzo de 1957, aparece en su artículo 128: «A propuesta de la Comisión y previa consulta al Comité económico y social, el Consejo establecerá los principios generales para la ejecución de una política común de formación profesional, capaz de contribuir al desarrollo armonioso de las economías nacionales y del mercado común». Se explicita el intento de crear una política común en cuanto a la formación profesional, idea que ha ido derivando hacia una cooperación y trabajo común, como queda reflejado en Maastricht y posteriormente en Amsterdam.

En los años 1963 y 1974 aparecen iniciativas en las que se legisla acerca del reconocimiento mutuo de diplomas, certificados y otros títulos de cualificaciones formales: "La política común de formación profesional deberá orientarse de tal forma que permita la aproximación progresiva de los niveles de cualificación». Se enuncia igualmente que en colaboración con los Estados miembros, la Comisión redactará, según las necesidades, y con relación a las distintas profesiones que exijan una formación específica, una descripción armónica de las cualificaciones básicas requeridas para el acceso a los diferentes niveles de formación.

\subsection{La década de los 80: un intento de política común}

En estos años aparecen diversas iniciativas en el ámbito de la formación profesional, con programas que fomentan el intercambio de estudiantes y trabajadores, la colaboración entre centros de enseñanza y empresas, la igualdad de oportunidades, el desarrollo de cualificaciones en torno a las nuevas tecnologías y la enseñanza de idiomas. En 1983 se establecen los cinco niveles de cualificación, niveles que se enuncian más adelante y en 1985 se facilita la movilidad laboral dentro de la comunidad, desarrollando cualificaciones profesionales, compatibles entre los estados miembros.

\footnotetext{
${ }^{5}$ (OJ 1963:63 / OJ 1974:14)
} 
La "Carta comunitaria de los derechos sociales fundamentales de los trabajadores» (1989) ${ }^{6}$, adoptada por los Jefes de Gobierno de once Estados miembros de la Comunidad Europea en el Consejo Europeo de Estrasburgo, el día 9 de diciembre de 1989, establece en el punto 15 que: «Todo trabajador de la Comunidad Europea debe poder tener acceso a la formación profesional y poder beneficiarse de la misma a lo largo de su vida activa. En las condiciones de acceso a dicha formación no podrá darse ninguna discriminación basada en la nacionalidad. Las autoridades públicas competentes, las empresas o los interlocutores sociales, cada uno en el ámbito de su competencia, deberían establecer los mecanismos de formación continuada y permanente que permitan a toda persona reciclarse, en particular mediante permisos de formación, perfeccionarse y adquirir nuevos conocimientos, teniendo en cuenta, particularmente, la evolución técnica».

Es una década marcada por los principios generales establecidos en 1963 y las iniciativas de 1974. Un intento de establecer políticas comunes que no obtuvo el resultado que se esperaba.

\subsection{Los años 90 marcados por el principio de subsidiariedad}

Será en esta década cuando se asuma el denominado principio de subsidiariedad y se dé un cambio de rumbo en la estrategia política de la Unión con respecto a la formación profesional. Se destaca el Tratado de Maastricht, que en su artículo $127^{7}$, expresa la intención y la competencia de la Comunidad en este ámbito, donde la armonización de los sistemas de formación profesional a través de instrumentos legales de la UE, queda excluida por el principio de subsidiariedad que estipula el mencionado artículo 127 del Tratado (La Unión debe apoyar y complementar las políticas de Formación Profesional de los Estados). Los sistemas nacionales «convergerán», entonces, por las vías de la presión de la competitividad internacional y las tecnologías. Esta política queda reafirmada nuevamente en el Tratado de Amsterdam, donde el artículo referido a la Educación y Formación Profesional es el 150:

1. La Comunidad desarrollará una política de formación profesional que refuerce y complete las acciones de los Estados miembros, respetando plenamente la responsabilidad de los mismos en lo relativo al contenido y a la organización de dicha formación.

2. La acción de la Comunidad se encaminará a:

- facilitar la adaptación a las transformaciones industriales, especialmente mediante la formación y la reconversión profesionales;

\footnotetext{
${ }^{6}$ Los Estados miembros se dotaron de una carta magna de lo social que aprobada en 1989, con la no adhesión de Gran Bretaña y desde 1998 asumida por todos los Estados, estableciendo los siguientes derechos:

- Derecho a la libre circulación

- Derecho al empleo y a la remuneración.

- Mejora de las condiciones de vida y de trabajo.

- Derecho a la protección social

- Derecho a la libertad de asociación y a la negociación colectiva

- Derecho a la formación profesional

- Derecho a la igualdad de trato entre hombres y mujeres.

- Derecho a la información, consulta y participación de los trabajadores

- Derecho a la protección de la salud y de la seguridad en el lugar de trabajo.

- Derecho a la protección de los niños y de los adolescentes.

- Derechos de las personas de edad.

- Derechos de las personas minusválidas.

7 Tratado de la Unión Europea, firmado el 7 de febrero de 1992 en Maastricht.
} 
- mejorar la formación profesional inicial y permanente, para facilitar la inserción y la reinserción profesional en el mercado laboral;

- facilitar el acceso a la formación profesional y favorecer la movilidad de los educadores y de las personas en formación, especialmente de los jóvenes;

- estimular la cooperación en materia de formación entre centros de enseñanza y empresas, e

- incrementar el intercambio de información y de experiencias sobre las cuestiones comunes a los sistemas de formación de los Estados miembros.

3. La Comunidad y los Estados miembros favorecerán la cooperación con terceros países y con las organizaciones internacionales competentes en materia de formación profesional.

4. El Consejo, con arreglo al procedimiento previsto en el artículo $189 \mathrm{C}$ y previa consulta al Comité Económico y Social, adoptará medidas para contribuir a la realización de los objetivos establecidos en el presente artículo, con exclusión de toda armonización de las disposiciones legales y reglamentarias de los Estados miembros. (Artículo 127de Maastricht y Artículo 150 de Amsterdam).

Atendiendo a estas líneas establecidas en Maastricht, se realiza un esfuerzo por parte de la Unión que queda reflejado en la Resolución del Consejo de 11 de junio de $1993^{8}$ relativa a la formación profesional para los años noventa, en la que se establecen las siguientes consideraciones:

- Considera que debe mejorarse la calidad de la formación profesional en cuanto a mejorar las posibilidades de desarrollo del individuo, ofrecer a todos los jóvenes una formación profesional reconocida y básica, asegurar el atractivo del sistema de formación profesional, utilizar la formación profesional como un instrumento para evitar la exclusión social y tener en cuenta las necesidades de la economía y competitividad de las empresas.

- Considera que deberían tenerse en cuenta las tendencias más importantes en los años noventa, fomentando las estrategias educativas que integren el aprendizaje general y la formación práctica, ya que la formación profesional tendrá que facilitar nuevas tecnologías, otras formas de organización y nuevos métodos de trabajo, promoviendo vínculos entre la oferta de formación básica y la formación continuada, introduciendo nuevas tecnologías que fomenten el desarrollo de nuevas cualificaciones y poniendo un mayor énfasis en la formación como elemento básico de la estrategia de las empresas.

- Pone de relieve el esfuerzo por crear una estrecha relación entre la formación profesional, la expedición de titulaciones de gran calidad y la lucha contra el desempleo.

- Considera que la formación tiene un papel que desempeñar en las pequeñas y medianas empresas.

- Considera importante que los interlocutores sociales participen en la definición de los fines y contenidos de los programas de formación.

- Subraya que los programas de los Estados miembros deberían incluir objetivos tales como el fomento de inversiones en formación profesional; el incremento de los vínculos entre la formación profesional de tipo académico y la basada en el sector laboral, ofrecer mayores oportunidades para los jóvenes y los adultos menos aventajados; aumentar la cooperación entre las autoridades locales y nacionales, los empresarios y los sindicatos, las empresas y otros organismos; satisfacer las nuevas necesidades en titulaciones futuras y aumentar la

8 Resolución del Consejo de 11 de junio de 1993 relativa a la formación profesional para los años noventa. DO.N. ${ }^{\circ}$ C 186 de 8.7.1993, p. 3. 
movilidad de los trabajadores, mejorar la formación del personal docente, mejorar los servicios de orientación, promover mayor innovación y más investigación aplicada, al igual que mayor dimensión europea en la formación inicial y continuada.

- Insta a la Comisión a que se trabaje en pro de una mayor coherencia a nivel comunitario entre los programas de formación y de enseñanza profesional, se fomente la cooperación europea en materia de titulaciones, se aumente la cooperación a escala transnacional, y todo ello aumentando el número de programas transnacionales y fomentando la transferencia de innovaciones y el desarrollo de la investigación aplicada.

Igualmente, el Consejo Europeo reunido en Essen los días 9 y 10 de diciembre de 1994 afirma que la lucha contra el desempleo y la igualdad de oportunidades entre hombres y mujeres seguirá siendo en el futuro la principal tarea de la Unión Europea y sus Estados miembros. Entre las medidas que habría que adoptar en cinco ámbitos, los dos primeros son los que hacen referencia a la necesidad de fomentar las inversiones en la formación profesional, «el mayor número de personas posible debería recibir una formación profesional y continua, en forma de aprendizaje a lo largo de la vida para poder adaptarse a los cambios del progreso tecnológico y limitar así el riesgo de perder su puesto de trabajo».

El Consejo Europeo de Cannes, celebrado los días 26 y 27 de junio de 1995, que reúne por primera vez a los 15 Estados miembros afirma que «deben reforzarse las políticas de formación y aprendizaje, elementos fundamentales para la mejora del empleo y la competitividad, y en especial la formación permanente». El Consejo Europeo tomó nota de la intención de la Comisión de presentar un Libro Blanco sobre formación antes de finalizar el año 95.

\subsection{Libro Blanco sobre educación y formación9}

En noviembre de 1995 se publica el Libro Blanco sobre la Educación y la Formación, en el que se recogen aportaciones de todos los países de la Unión Europea y se plantea un modelo y unas tendencias en este ámbito que se pueden considerar comunes. A continuación se plantean algunas reflexiones y aportaciones que aparecen en este documento elaborado por la Comisión de las Comunidades Europeas y que ha titulado Enseñar y aprender: Hacia la Sociedad Cognitiva ${ }^{10}$.

Se consideran tres las presiones fundamentales existentes hoy en nuestro contexto que están influyendo significativamente en el planteamiento y desarrollo de nuestros sistemas de educación y formación:

1. La difusión masiva de las tecnologías de la información y la comunicación han transformado la naturaleza del trabajo y la organización de la producción, por lo que el trabajo tendrá un contenido cada vez más cargado de tareas inteligentes que requieran iniciativa y adaptación.

\footnotetext{
${ }^{9}$ El día 29 de noviembre de 1995 la comisión Europea adopta el Libro Blanco sobre educación y formación.

10 Conclusiones del Consejo de 6 de mayo de 1996 sobre el Libro blanco Enseñar y Aprender: hacia la sociedad cognitiva. DO.N. ${ }^{\circ} \mathrm{C}$ 195 de 6.7.1996. p. 1
} 
2. La presión del mercado mundial significa que cualquier empresa podrá realizar transacciones económicas con cualquier país, derivando, posiblemente, procesos de producción a otros países con diferentes costes.

3. La renovación científica y técnica acelerada impedirá que las personas realicen tareas y procesos que la nueva tecnología realizará de forma automática, lo que significa que los trabajadores deberán utilizar más sus habilidades cognitivas para incorporarse a los procesos de producción.

Esta nueva situación obliga a plantearse un sistema de respuesta y anticipación a los cambios producidos. La Comisión, con las aportaciones de todos los países miembros, presenta las siguientes conclusiones en este Libro Blanco:

- Los sistemas educativos deberán centrarse en el desarrollo de la aptitud para el empleo y la actividad que exige determinar las aptitudes requeridas, tales como conocimientos fundamentales y básicos, conocimientos técnicos, aptitudes sociales, y cómo adquirirlas.

- Para asegurar la adquisición de aprendizajes es necesario la creación de redes en las que participen todos los agentes implicados y con responsabilidad en el proceso, la cooperación entre escuela/familia, para garantizar la adquisición de conocimientos básicos y entre los centros de enseñanza y las entidades empresariales, para garantizar la adquisición de competencias clave, técnicas y comportamentales.

- El concepto de «competencias clave» se generaliza en el conjunto de formaciones, siendo necesario establecer los mejores medios para adquirirlas, evaluarlas y acreditarlas.

- Aparece como cuestión central la relación entre educación y formación profesional, siendo uno de los grandes retos de los sistemas educativos el disponer de un sistema de formación profesional que responda con eficacia y calidad a los requerimientos del entorno productivo y a las nuevas exigencias del marco de relaciones laborales y profesionales establecidas.

- La vinculación entre la escuela y la empresa y las prácticas y sistemas de alternancia, supone el desarrollo de módulos de formación en centros de trabajo, exigiendo la participación activa de los centros formativos y las entidades empresariales, para desarrollar con eficacia y rentabilizar estos procesos formativos.

- Los dispositivos de "garantía de formación», destinados a los colectivos que abandonan el sistema de formación y cualificación, sin adquirir alguna competencia que permita la incorporación al mercado de trabajo.

- Es preciso el diseño de un sistema de acreditación, validación y reconocimiento de competencias a nivel europeo, que podría concretarse en un proyecto de «tarjetas personales de competencias».

- Todos los países europeos tienen diseñado un modelo de formación permanente o continua, que permite a la población trabajadora adaptarse, mediante procesos formativos, a las nuevas exigencias tecnológicas de producción y a los cambios producidos en el entorno de las relaciones laborales.

- Nuevas fórmulas de financiación de la educación y la formación, en las que la nueva disposición y necesidades planteadas en el marco de la formación exigen la participación 
económica y financiera de aquellos que reciben la formación y de quienes se benefician de una población mejor formada y más cualificada.

- La creación de un observatorio de prácticas innovadoras en materia de formación profesional para definir y mantener sistemas de calidad de los sistemas educativos que nos permitan medir el impacto y el alcance de las acciones educativas y formativas desarrolladas.

- El reconocimiento mutuo de módulos de formación, con una definición de familias profesionales, perfiles profesionales, profesiones, puestos de trabajo ajustada a las necesidades y exigencias del entorno empresarial y productivo, que nos lleve a un diseño adecuado de acciones y procesos formativos y de cualificación profesional con respuesta directa a dichas necesidades presentes y futuras.

Esta situación lleva a determinar algunos «objetivos prioritarios», que es necesario conseguir en todos los países miembros de la Unión Europea ${ }^{11}$ :

- Fomentar la adquisición de nuevos conocimientos.

- Acercar la escuela a la empresa.

- Luchar contra la exclusión.

- Hablar tres lenguas comunitarias.

- Tratar en un plano de igualdad la inserción en equipamientos y la inversión en formación.

- Abrir la educación al mundo del trabajo.

- Implicar a la empresa en el esfuerzo de formación.

- Fomentar la cooperación entre centros de enseñanza y empresas.

- Fomentar a escala europea la figura del aprendiz.

- Fomentar la formación para nuevos oficios del sector de servicios, insistiendo en el carácter multidisciplinario: turismo, protección del medio ambiente.

- Apoyar la formación de ingenieros y técnicos del sector terciario.

- Fomentar la formación para la creación de empresas.

- Crear un observatorio europeo de las prácticas innovadoras de formación profesional.

- Crear dispositivos de anticipación de las necesidades de competencias, cualificaciones y nuevos oficios.

Otro aspecto a tener en cuenta es la decisión del Parlamento Europeo y del Consejo por la que se estableció el año 1996 como el Año Europeo de la Educación y la Formación, que se definió como «Oportunidades educativas y formativas para el desarrollo personal y profesional a lo largo de toda la vida». Los objetivos de este año europeo han sido sensibilizar a los europeos sobre el concepto de educación y formación permanente y llevar a cabo, a través de distintas iniciativas a varios niveles (europeo, nacional, regional y local), una reflexión de conjunto sobre el papel y la importancia de la educación y la formación en el siglo XXI.

11 Conclusiones del Consejo de 24 de julio de 1995 relativas a la importancia y a los retos de la formación profesional. DO.N. ${ }^{\circ}$ C 207 de 12.8.1995, p. 7 . 


\subsection{Una política de empleo en la Unión Europea}

La Comunidad no tiene una política de empleo, sino un conjunto de acciones relativas al empleo y múltiples recomendaciones referidas al mercado de trabajo. En la normativa comunitaria, en concreto en la introducción del título VIII del Tratado de Amsterdam se establece que:

- Anualmente se elaborarán unas directrices de empleo (derivadas de la Cumbre de Luxemburgo en noviembre de 1997), que deben ser incorporadas por cada uno de los Estados miembros en sus planes nacionales de empleo, que son presentados a finales de mes de mayo de cada año ante la Comisión.

- Las directrices de empleo hacen referencia a cuatro pilares básicos, como son la mejora de la capacidad de inserción profesional o empleabilidad, el fomento del espíritu de empresa, la adaptabilidad de trabajadores y empresas y la igualdad de oportunidades.

- La Comisión y los Estados miembros analizan las planes nacionales de empleo y emiten sus recomendaciones.

- El Consejo, apoyándose en los Informes de la Comisión sobre el empleo y en las recomendaciones aportadas elabora unas directrices.

Aunque no existe una política económica de empleo, la Comunidad creó el Fondo Social Europeo (FSE), reformado en varias ocasiones en función de la evolución económica y social. Este fondo proporciona ayudas para cualquier tipo de actuación que esté vinculada directamente al empleo y sirva para potenciarlo. Hay que constatar que entre el 80 y el $90 \%$ de las acciones financiadas por este fondo son en materia de formación profesional, destacando que las acciones formativas deben tener una inmediata relación con el empleo.

La gran novedad del Tratado de Amsterdam es que la comunidad asume como objetivos básicos el formato del empleo, la mejora de las condiciones de vida y de trabajo, la protección social, el diálogo social y el desarrollo de los recursos humanos. La estrategia política de empleo en la UE es compleja y se articula sobre diferentes variables orientadas a incidir en las características estructurales de la oferta de trabajo, como son el desarrollo de un sistema de cualificación profesional, el establecimiento de un marco institucional de políticas activas de empleo, la promoción de la igualdad de oportunidades y la flexibilidad laboral), al igual que en el incentivo de la demanda de empleo en un marco institucional que apuesta por el método de concertación, la negociación colectiva y la participación sindical.

La Unión Europea traza cuatro bloques temáticos en los que deben centrarse las políticas de empleo de los Estados miembros.

- Adopción de políticas activas de empleo.

- Políticas de formación profesional o de recualificación profesional permanentes.

- Marcos jurídicos laborales, fiscales y administrativos.

- Flexibilidad en la organización del trabajo. 
- Políticas de igualdad de oportunidades.

El primer bloque de directrices para el empleo es «mejorar la capacidad de inserción profesional», en el cual el concepto clave es la «empleabilidad», que en el ámbito comunitario adopta un enfoque donde prevalece la atención a los factores sociales que condicionan la posición en el mercado de trabajo y no la condición subjetiva o personal, en el diagnóstico de la situación de empleo.

El desarrollo de Europa como potencia económica mundial debe buscarse en el desarrollo tecnológico, en la aplicación de políticas macroeconómicas estables y tendencialmente expansivas, y en su capacidad de favorecer el desarrollo y la reproducción de capitales humanos específicos, los únicos capaces de sacar partido eficazmente de las tecnologías, la ampliación del mercado y la estabilidad monetaria.

Un dato preocupante es que el nivel de participación de los trabajadores en cursos de formación ocupacional es bastante más elevado entre los trabajadores con un cierto nivel de cualificación que entre los trabajadores con baja cualificación; esta tendencia parece cada vez mayor a consecuencia de las cualificaciones requeridas por los nuevos empleos vinculados a las sociedad de la información ${ }^{12}$.

Los días 23 y 24 de marzo del 2002 la celebración de un consejo europeo especial en Lisboa, para adoptar la nueva estrategia de la Unión en materia de empleo, reforma económica y cohesión social como partes integrantes de una economía basada en el conocimiento.

\section{RECONOCIMIENTO DE TÍTULOS Y SISTEMA DE CORRESPONDENCIA DE CUALIFICACIONES EN LA UNIÓN EUROPEA}

La educación y la formación juegan un papel fundamental en el desarrollo económico y cultural de la sociedad europea y en los últimos años los Estados miembros de la Unión Europea han logrado un amplio consenso sobre el hecho de que el capital humano es uno de los recursos vitales. Por ello las instituciones comunitarias están potenciando el desarrollo de la educación y la formación profesional.

La libre circulación aparece como uno de los objetivos centrales de la Unión Europea y debe quedar garantizada tanto para los trabajadores asalariados, como para aquellos que realizan actividades independientes en un Estado miembro distinto de aquel en el que la persona tiene fijada su residencia, bien sea mediante el establecimiento en el Estado miembro en el que desea desarrollar la actividad en cuestión, o bien limitándose a la mera prestación de servicios.

Para facilitar el ejercicio de actividades en el conjunto de los Estados miembros se hace necesario que la persona esté en posesión de un título probatorio de la posesión de los conocimientos adecuados para el ejercicio de la profesión, y que dicho título sea reconocido por las autoridades del Estado miembro

\footnotetext{
${ }^{12}$ El día 24 de julio de 1996 la Comisión Europea adopta un libro verde titulado Vivir y trabajar en la sociedad de la información:
} 
en que desea llevar a cabo su actividad. La Unión europea se ha preocupado desde sus orígenes, de organizar el reconocimiento mutuo de estas condiciones de acceso a la profesión ${ }^{13}$.

En este sentido, se han ido elaborando una serie de directivas que tienen fuerza vinculante con respecto a los Estados por lo que se refiere al resultado que hay que alcanzar, pero dan libertad a los Estados para elegir la forma y los medios para su ejecución. En caso de que se plantease un conflicto entre una directiva y una disposición nacional, prevalecerá la primera y las personas interesadas así podrán hacerlo valer ante los tribunales nacionales. ${ }^{14}$

En un principio, la Unión Europea elaboró, profesión por profesión, numerosas directivas cuyo contenido varía según los tipos de actividad. Para la mayoría de las actividades industriales, artesanales y comerciales se han adoptado directivas de liberalización, reconociéndose en el país de acogida la experiencia profesional adquirida en el país de origen.

Existe asimismo una Directiva del Consejo de 18 de junio de 1992 relativa a los nacionales de los Estados miembros que poseen un título o un certificado en un país tercero ${ }^{15}$. En ella se invita a los Gobiernos de los Estados miembros a que faciliten a estas personas el acceso al ejercicio de las profesiones reguladas dentro de la Unión Europea, reconociendo dichos títulos, certificados y otras titulaciones en su territorio.

La Decisión del Consejo de 16 de julio de 1985 relativa a la correspondencia de las cualificaciones de formación profesional entre los Estados miembros de la Comunidades Europeas tiene como principal objetivo favorecer la libre circulación de los trabajadores ${ }^{16}$ y la creación de las condiciones que permitan la igualdad de acceso al empleo.

Para lograrlo se establece un sistema encargado de ofrecer informaciones en materia de correspondencia de las cualificaciones de formación profesional para ayudar al trabajador migrante a dar a conocer mejor sus cualificaciones a la hora de acceder a un empleo en otro Estado miembro.

Al tratarse de datos de naturaleza técnica no comportan obligación jurídica para el Estado miembro de acogida sino que favorecen la comprensión recíproca entre empresario y trabajador y la actividad institucional de mediación de los servicios públicos de empleo.

El objetivo de estos datos no es alcanzar un reconocimiento jurídico de los diplomas, certificados y otros títulos de formación profesional. Este reconocimiento jurídico no es necesario más que para el acceso

\footnotetext{
prioridad para las personas.

${ }^{13}$ CEDEFOP (1992): El proyecto comunitario. Correspondencia de las cualificaciones de formación profesional; objetivos, método de trabajo, evaluación. Berlín.

14 Resolución del Consejo de 3 de diciembre de 1992 sobre la transparencia de las cualificaciones profesionales. DO.N. ${ }^{\circ}$ C 49 de 19. 2.1993, p. 1.

15 Directiva 92/51/CEE del Consejo de 18 de junio de 1992 relativa a un segundo sistema general de reconocimiento de formaciones profesionales, que completa la directiva 89/48/CEE. DO L209 de 24/07/1992, p.25.

${ }^{16}$ «[...] todo ciudadano de un Estado miembro, sea cual fuere su residencia, tiene derecho a acceder a una actividad asalariada y de ejercerla en el territorio de otro Estado miembro, de acuerdo con las disposiciones legislativas, reglamentarias y administrativas que rigen el empleo de los trabajadores nacionales de este Estado». Art. 1 del Reglamento 1612/68 del Consejo, relativo a la libre circulación de los trabajadores en el interior de la Comunidad.
} 
a las profesiones reguladas, es decir aquellas que en el acceso queda subordinado, por medio de disposiciones jurídicas, reglamentarias o administrativas a la posesión de un diploma, un certificado u otro título de formación que pertenezca al sistema nacional de formación. De esta manera, mientras el reconocimiento de los diplomas tiene por objetivo certificar el valor legal de un título, el sistema de correspondencia de las cualificaciones trata de suministrar la información necesaria para que un trabajador migrante pueda dar a conocer y utilizar lo mejor posible el nivel y el contenido de sus cualificaciones de formación profesional.

Los estudios de correspondencia de la cualificaciones incluyen una descripción comunitaria de las exigencias profesionales prácticas y un cuadro comparativo de los diplomas, certificados y otros títulos de formación profesional expedidos en todos los Estados miembros.

El procedimiento seguido para el desarrollo de estos trabajos es el indicado en la Decisión del Consejo de 16 de julio de 1985. Su aplicación la llevó a cabo la Comisión con la ayuda técnica del CEDEFOP. Asimismo asiste a la Comisión un grupo de coordinadores nacionales.

Los trabajos de correspondencia de cualificaciones pueden utilizar como referencia la estructura de los niveles de formación elaborada por la Comisión con la ayuda del Comité Consultivo de la Formación Profesional. El texto de dicha estructura se incluye en la Decisión de 16 de julio de $1985^{(17)}$ y se divide en:

\begin{tabular}{|l|l|}
\hline NIVEL 1 & $\begin{array}{l}\text { Formación que da acceso a este nivel: escolaridad obligatoria } \\
\text { y preparación profesional. Una cualificación que apunta a } \\
\text { trabajos relativamente simples, los cuales pueden ser } \\
\text { adquiridos en un tiempo bastante corto. }\end{array}$ \\
\hline NIVEL 2 & $\begin{array}{l}\text { Formación que da acceso a este nivel: escolaridad obligatoria } \\
\text { y formación profesional (incluido en particular el aprendizaje). } \\
\text { Se orienta principalmente al trabajo práctico que pueda ser } \\
\text { desarrollado sin supervisión, al menos respecto a aquellas } \\
\text { técnicas que ha sido aprendidas previamente. }\end{array}$ \\
\hline NIVEL 3 & $\begin{array}{l}\text { Formación que da acceso a este nivel: escolaridad obligatoria } \\
\text { y/o formación profesional y formación técnica complementaria } \\
\text { o formación técnica escolar u otra de nivel secundario. }\end{array}$ \\
$\begin{array}{l}\text { Fundamentalmente trabajo práctico que puede ser realizado } \\
\text { sin supervisión y/o incluya otras responsabilidades tales como } \\
\text { las de planificación y coordinación. }\end{array}$ \\
\hline NIVEL 4 & $\begin{array}{l}\text { Formación que da acceso a este nivel: formación secundaria } \\
\text { (general o profesional) y formación técnica post-secundaria. } \\
\text { Trabajo generalmente autónomo de forma independiente para } \\
\text { la planificación conceptual y/o administrativa y/o gerencial. }\end{array}$ \\
\hline NIVEL 5 & $\begin{array}{l}\text { Formación que da acceso a este nivel: formación secundaria } \\
\text { (general o profesional) y formación superior completa. } \\
\text { Posibilita el ejercicio de la práctica profesional si el asalariado } \\
\text { o autoempleo demuestra independientemente dominio de las } \\
\text { bases científicas de la profesión. }\end{array}$ \\
\hline
\end{tabular}

- Con el fin de dar a conocer mejor sus cualificaciones, el trabajador migrante puede solicitar a los servicios competentes de su país antes de trasladarse, o del país de acogida si ya lo ha

17 Décision du conseil du 16 juillet 1985 concernant la correspondance des qualifications de formation professionnelle entre Etats membres des Communautés européennes. JO.N. ${ }^{\circ}$ L 199. du 31.7.85, p. 56. 
hecho, una ficha de información en la que se incluye la descripción comunitaria y el cuadro comparativo relativa a esa profesión.

- Por su parte y para la realización de los trabajos científicos y técnicos necesarios para la elaboración de las descripciones comunitarias y los cuadros comparativos, la Comisión cuenta con la ayuda del Centro Europeo para el desarrollo de la formación profesional (CEDEFOP) con sede en Tesalónica actualmente.

- En España las autoridades competentes en materia de correspondencia de cualificaciones son el Ministerio de Educación y Ciencia (Subdirección General de Ordenación Académica) y el Ministerio de Trabajo y Seguridad Social (Instituto Nacional de Empleo, Subdirección General de Gestión de Formación Ocupacional).

- Desde la perspectiva europea el tema de la transparencia del mercado y de las cualificaciones se abordó parcialmente mediante las Directivas 89/48/CEE, relativa al reconocimiento de títulos de enseñanza superior y 02/51/CEE, sobre profesiones reguladas, ambas transpuestas al ordenamiento español por los Reales Decretos 1665/1991, de 15 de octubre y 1396/1995, de 4 de agosto.

Los proyectos de la Comisión son:

- El portafolio o cartera individual de cualificaciones, lanzado en 1993 con la pretensión de diseñar un «curriculum vitae europeo» normalizado ${ }^{18}$.

- La tarjeta o carné personal de competencias, vinculado a un sistema europeo de acreditación de competencias.

\section{INSTRUMENTOS Y FINANCIACIÓN PARA LOGRAR CRITERIOS DE HOMOGENEIDAD Y UNIDAD}

\subsection{El Fondo Social Europeo (FSE): Objetivos}

El primer antecedente del Fondo Social Europeo (FSE) se encuentra en el Tratado de París de 1951, por el que se crea la Comunidad Económica del Carbón y del Acero (CECA). En dicho tratado se estableció un fondo de ayuda al reempleo, la recolocación y la reeducación profesional de los trabajadores que perdían su empleo como consecuencia directa de la creación del mercado común del acero y del carbón. Su objeto era atenuar los negativos efectos sociales que se iban a producir como consecuencia del establecimiento de la CECA. El tratado de Roma, constitutivo de la CEE, establece en su artículo 123 y siguientes, un Fondo para «promover dentro de la comunidad las oportunidades de empleo y la movilidad geográfica y profesional de los trabajadores».

\footnotetext{
${ }^{18}$ Decisión del Consejo del 21 de diciembre de 1998 relativa a la promoción de itinerarios europeos de formación en alternancia,
} incluido el aprendizaje, por el que se crea el Europass-formación. 
El artículo 146 del Tratado de la Unión Europea, firmado el 7 de febrero de 1992, en Maastricht, modifica el artículo 123 del tratado de Roma, ampliando el cometido del FSE a fin de facilitar la adaptación de los trabajadores a las transformaciones industriales y a los cambios de los sistemas de producción, especialmente mediante la formación y la reconversión profesional. En la historia del FSE se pueden distinguir seis etapas:

- 1958/1970: En un contexto de crecimiento económico y pleno empleo en el conjunto de los seis Estados miembros, con la excepción del sur de Italia, el FSE favorecía el reciclaje y la movilidad profesional de los ocupados en ciertos sectores en reconversión y la reinserción profesional de ciertos colectivos de parados, subempleados y personas con discapacidad. Los recursos del FSE se asignaban bajo la fórmula del reembolso de gastos ya realizados por los Estados miembros.

- 1971/1983: La economía europea, bajo los efectos de las «crisis del petróleo» y el cambio estructural, empieza a sufrir importantes desequilibrios en sus mercados de trabajo. EI FSE, en este contexto, es objeto de una profunda reforma para convertirse en un importante instrumento de política comunitaria. Si bien la tipología de las acciones y los colectivos beneficiarios no se modifican profundamente, los mecanismos de gestión se transforman sustancialmente, acercándolos al modelo vigente.

- 1984/1989: La integración en la CEE de Irlanda, Grecia, España y Portugal acentúa las diferencias de renta entre las regiones que componen la comunidad. Lo más relevante de esta etapa es la tímida aplicación de criterios de cohesión regional en la utilización del Fondo. El $40 \%$ de las intervenciones se concentraba en las regiones más atrasadas. Las restantes ayudas iban a zonas de paro elevado y de larga duración y/o reestructuración industrial y sectorial. Las ayudas del FSE se dirigían prioritariamente a los jóvenes menores de 25 años y a los adultos en paro de larga duración, amenazados de paro, mujeres, personas con discapacidad, migrantes y ocupados en PYME.

- 1990/1993: Está marcada por la reforma de los fondos estructurales en 1988. La idea básica de esta reforma, la más importante realizada hasta la fecha, es la integración de los fondos, conservando la independencia de gestión de cada uno de ellos, en la política comunitaria dirigida a reforzar la solidaridad y cohesión económica y social entre las regiones más atrasadas y más desarrolladas de la comunidad.

- 1994/1999: En 1993 se produjo una adaptación de la normativa reguladora de los fondos estructurales en base a lo previsto en el artículo 19 del Reglamento (CEE) 2052/88. Esta revisión reglamentaria no modificó los aspectos esenciales de la reforma del año 1988. Los principios sobre los que se apoyó no sólo se mantuvieron, sino que se reforzaron.

- 2000/2006: La misión del FSE en este período es la de apoyar medidas de prevención y lucha contra el desempleo, de desarrollo de los recursos humanos y de integración social en el medio laboral a fin de promover un elevado nivel de empleo, la igualdad entre hombres y mujeres, un desarrollo sostenible y la cohesión económico y social. En especial, contribuirá a las acciones emprendidas en virtud de la estrategia europea de empleo y de las directrices anuales sobre el 
empleo, recogidas en los planes nacionales de acción para el empleo que diseñan los Estados miembros ${ }^{19}$.

El instrumento fundamental de la política social de la Unión Europea es el Fondo Social Europeo ${ }^{20}$, atribuyéndole la función de fomentar en la Comunidad las oportunidades de empleo y la movilidad geográfica y profesional de los trabajadores, luchando contra el desempleo; facilitando el acceso al mundo del trabajo; fomentando la igualdad de oportunidades en el mercado laboral; desarrollando las competencias, aptitudes y cualificaciones profesionales, y ayudando a la creación de nuevos puestos de trabajo.

Está acompañado de otros dos fondos ${ }^{21}$, el Fondo Europeo de Desarrollo Regional (FEDER) que pretende reducir las diferencias de desarrollo entre las regiones de la Unión; el Fondo Europeo de Orientación y Garantía Agrícola (FEOGA), que contribuye a la adaptación de las estructuras agrarias y al desarrollo y diversificación de las zonas rurales. Estos tres instrumentos constituyen los Fondos Estructurales de la Unión Europea. A estos se ha añadido el Instrumento Financiero de Orientación de la Pesca (IFOP), que apoya la reestructuración del sector pesquero.

Con 8 millones de parados de larga duración y muchos jóvenes que carecen de las cualificaciones requeridas por el mercado de trabajo, el FSE se ha centrado en programas dirigidos a estos colectivos, otros beneficiarios son los trabajadores amenazados por el desempleo, las mujeres, los inmigrantes y los trabajadores de las pequeñas y medianas empresas.

De conformidad con la normativa reguladora de los fondos estructurales los objetivos de estos fondos han sido hasta finales de 1999 los siguientes:

\begin{tabular}{|l|l|}
\hline Objetivo 1 & $\begin{array}{l}\text { Fomentar el desarrollo y el ajuste estructural de las } \\
\text { regiones menos favorecidas. }\end{array}$ \\
\hline Objetivo 2 & $\begin{array}{l}\text { Reconvertir las regiones gravemente afectadas por el } \\
\text { declive industrial. }\end{array}$ \\
\hline Objetivo 3 & $\begin{array}{l}\text { Facilitar la inserción de las personas expuestas a la } \\
\text { exclusión del mercado laboral. }\end{array}$ \\
\hline Objetivo 4 & $\begin{array}{l}\text { Facilitar la adaptación de los trabajadores a las } \\
\text { mutaciones industriales y a la evolución de los sistemas de } \\
\text { producción. }\end{array}$ \\
\hline Objetivo 5 & $\begin{array}{l}\text { 5a) Acelerar la adaptación de las estructuras agrarias, la } \\
\text { ayuda a la modernización y la reestructuración de la } \\
\text { pesca. } \\
\text { 5b) Facilitar el desarrollo y el ajuste estructural de las } \\
\text { zonas rurales. }\end{array}$ \\
\hline
\end{tabular}

\footnotetext{
${ }^{19}$ EI FSE en esta etapa está regulado mediante el Reglamento (CE)N. ${ }^{\circ}$ 1784/1999 del Parlamento Europeo y del Consejo de 12 de julio de 1999.

${ }^{20}$ Los artículos 117 y118 del Tratado de Roma de 25 de marzo de 1957, constitutivo de la Comunidad Económica Europea, recogen el acuerdo de los Estados miembros de promover la mejora de las condiciones de vida y de trabajo de la mano de obra, a fin de conseguir su equiparación y su progreso, encargando a la Comisión la misión de promover una estrecha colaboración entre los estados miembros en el ámbito social, particularmente en materias relacionadas, entre otras, con el empleo, la formación y el perfeccionamiento profesional. Reglamento CEE 2084/93 del Consejo.

${ }_{21}$ Los fondos comunitarios se crearon con el fin de reducir las diferencias entre los distintos niveles de desarrollo de los Estados miembros de la Unión europea. Nacieron y se desarrollaron como consecuencia del Tratado de la Comunidad Económica Europea, firmado en Roma en 1957 y el Tratado de la Unión Europea, firmado en Maastricht en 1992. Estos fondos son el Fondo de Desarrollo Regional (FEDER), el Fondo Social Europeo (FSE), el Fondo Europeo de Orientación y Garantía Agraria (FEOGA) y el Instrumento Financiero de Orientación y Pesca (IFOP).
} 
\begin{tabular}{|l|l|}
\hline Objetivo 6 & Fomentar el desarrollo en las regiones con niveles muy \\
\hline
\end{tabular} bajos de densidad.

Los nuevos objetivos para los fondos estructurales marcados por la Unión Europea para el próximo período 2000/2006 son los siguientes:

- Objetivo 122: Promover el desarrollo y el ajuste estructural de las regiones menos desarrolladas. Se financiará con los fondos FEDER, FSE, Orientación FEOGA, IFOP. Las regiones de Objetivo 1 son las zonas cuyo producto interior bruto (PIB) sea inferior al $75 \%$ de la media comunitaria. Quedan incluidas las zonas ultraperiféricas y las que se encontraban dentro de Objetivo 6.

- Objetivo 23: Apoyar la reconversión económica y social de las zonas con deficiencias estructurales. Se financiará con los fondos FEDER, FSE, Garantía FEOGA. Las zonas de objetivo 2 son aquellas en las que convenga apoyar la reconversión económica y social; zonas que estén experimentando transformaciones socioeconómicas en los sectores de la industria y los servicios, zonas rurales en declive; zonas urbanas con situación difícil: zonas pesqueras en crisis, siempre y cuando no afecte a más del $18 \%$ de la población total de la comunidad.

- Objetivo 324: Apoyar la adaptación y modernización de las políticas y sistemas de educación, formación y empleo. Se financiará con el Fondo Social Europeo.

Para este período 2000/2006 el FSE establece que apoyará y complementará las actividades de los Estados miembros destinadas al desarrollo del mercado de trabajo y de los recursos humanos en el marco de sus planes de acción nacionales para el empleo dentro de los siguientes ámbitos ${ }^{25}$ :

- Desarrollo y promoción de las políticas activas del mercado de trabajo

- Promoción de la igualdad de oportunidades para todos en el acceso al mercado de trabajo.

- Fomento y mejora de la formación profesional y del asesoramiento.

- Promoción de la mano de obra cualificada.

- Mejora del acceso y la participación de la mujer en el mercado de trabajo.

Las actuaciones a realizar en España en materia de recursos humanos, cofinanciados por el FSE durante el período 2000/2006, están dirigidas a alcanzar los siguientes objetivos:

- Incrementar los niveles de actividad y de empleo de la población y de los jóvenes en particular y disminuir la incidencia del desempleo de larga duración

- Favorecer la ocupación en PYME y en las distintas formas de economía social.

\footnotetext{
${ }^{22}$ Son zonas de objetivo 1 aquellas cuyo PIB es inferior al 75\% de la media comunitaria, en estas zonas participarán todos los fondos estructurales FEDER, FEOGA orientación; FSE, IFOP. En España son zonas de objetivo 1 todo el territorio de las siguientes comunidades y ciudades autónomas: Andalucía, Asturias, Canarias, Castilla-León, Castilla-La Mancha, Extremadura, Galicia, Murcia, Valencia, Ceuta, Melilla. La comunidad de Cantabria tiene una situación transitoria hasta el 31 de diciembre de 2205.

${ }^{23}$ Son zonas con problemas estructurales de reconversión económica y social. En España son zonas de Objetivo 2 parte del territorio de las Comunidades autónomas de Aragón, Baleares, Cataluña, Madrid, Navarra, País Vasco, La Rioja.

${ }^{24}$ Son zonas de objetivo 3 todo el territorio de la Unión Europea que esté fuera de objetivo 1. En estas zonas interviene únicamente el FSE.

${ }^{25} \mathrm{~A}$ la hora de desarrollar medidas derivadas de la aplicación de estos ámbitos se tendrá en cuenta la necesidad de apoyar el desarrollo local, incluidas las iniciativas de empleo locales y los pacto de empleo territoriales, la dimensión social y el capítulo del
} 
- Mejorar la capacidad de adaptación de los trabajadores y de las empresas a los cambios del sistema productivo.

- Perfeccionar el sistema de formación profesional de acuerdo con los requerimientos del mercado de trabajo.

- Incrementar la preparación del personal científico y la transferencia de tecnología hacia el sector productivo.

- Asegurar la equiparación entre géneros en el mercado de trabajo.

- Aumentar la participación de las personas con especiales dificultades en el mercado de trabajo.

- Intensificar la creación de empleo en el entorno local.

Para alcanzar los objetivos específicos en materia de recursos humanos fijados por el FSE para el período 2000/2006, se han programado actuaciones integradas en 9 ejes prioritarios y 22 medidas, que aparecen reflejadas en el cuadro siguiente:

\begin{tabular}{|c|c|}
\hline \begin{tabular}{ll|} 
Inserción & $y$ \\
reinserción &
\end{tabular} & $\begin{array}{l}\text { Ofrecer a los desempleados posibilidades de inserción en el } \\
\text { mercado laboral }\end{array}$ \\
\hline $\begin{array}{l}\text { ocupacional de } \\
\text { los }\end{array}$ & $\begin{array}{l}\quad \text { Combatir el paro prolongado mediante acciones de reinserción } \\
\text { laboral de los desempleados de larga duración }\end{array}$ \\
\hline desempleados & $>$ Ofrecer vías de inserción profesional a los jóvenes \\
\hline & $\begin{array}{l}\text { Apoyar la reincorporación a la vida laboral activa de las } \\
\text { personas ausentes del mercado de trabajo. }\end{array}$ \\
\hline $\begin{array}{l}\text { Refuerzo de la } \\
\text { capacidad } \\
\text { empresarial }\end{array}$ & $\begin{array}{l}\text { Favorecer la generación de nueva actividad que permita la } \\
\text { creación de empleo }\end{array}$ \\
\hline $\begin{array}{l}\text { Refuerzo de la } \\
\text { estabilidad en el } \\
\text { empleo }\end{array}$ & $\begin{array}{l}\begin{array}{l}\text { Asegurar la actualización del nivel de competencias de los } \\
\text { trabajadores. }\end{array} \\
\end{array}$ \\
\hline adaptabilidad & $>$ Sostener la consolidación del empleo existente. \\
\hline & $\begin{array}{l}\text { Fomentar los procesos de modernización de las } \\
\text { organizaciones públicas y privadas que favorezcan la creación } \\
\text { y la estabilidad en el empleo. }\end{array}$ \\
\hline & $\begin{array}{l}\text { Fomentar la permanencia de personas de edad madura en el } \\
\text { mercado laboral. }\end{array}$ \\
\hline $\begin{array}{l}\text { Refuerzo de la } \\
\text { educación } \\
\text { técnico } \\
\text { profesional }\end{array}$ & $\begin{array}{l}\text { Fomentar el acceso de todos a las enseñanzas de formación } \\
\text { profesional y su extensión, en sus dos componentes: } \\
\text { formación profesional de base y formación profesional } \\
\text { específica. }\end{array}$ \\
\hline & $\begin{array}{l}\text { Desarrollar nuevas modalidades de oferta en formación } \\
\text { profesional inicial/reglada }\end{array}$ \\
\hline & $\begin{array}{l}\text { Promover mecanismos de integración y mejora de la eficiencia } \\
\text { de los subsistemas de formación profesional. }\end{array}$ \\
\hline & $\begin{array}{l}\text { Proporcionar alternativas educativas enfocadas al mercado de } \\
\text { trabajo y a las personas que no superan la enseñanza } \\
\text { obligatoria }\end{array}$ \\
\hline $\begin{array}{ll}\begin{array}{l}\text { Refuerzo } \\
\text { potencial }\end{array} & \\
\text { humano } \\
\text { investigación, } \\
\text { ciencia } \\
\text { tecnología }\end{array}$ & $\begin{array}{l}\text { Apoyar la inversión en capital humano en el ámbito de la } \\
\text { investigación, la ciencia y la tecnología y la transferencia de } \\
\text { conocimientos hacia el sector productivo }\end{array}$ \\
\hline Participación de & $>$ \\
\hline
\end{tabular}

empleo en el seno de la sociedad de la información y la igualdad de oportunidades de mujeres y hombres. 


\begin{tabular}{|c|c|}
\hline \multirow{2}{*}{$\begin{array}{l}\text { las mujeres en } \\
\text { el mercado de } \\
\text { trabajo }\end{array}$} & Fomentar la actividad empresarial de las mujeres \\
\hline & $\begin{array}{l}\text { Combatir la segregación horizontal y vertical, así como la } \\
\text { discriminación salarial y favorecer la conciliación de la vida } \\
\text { familiar y laboral. }\end{array}$ \\
\hline \multirow{2}{*}{$\begin{array}{ll}\text { Integración en } \\
\text { el mercado de } \\
\text { trabajo de las } \\
\text { personas con } \\
\text { especiales } \\
\text { dificultades } \\
\end{array}$} & $\begin{array}{l}\text { Apoyar la inserción de las personas discapacitadas en el } \\
\text { mercado laboral. }\end{array}$ \\
\hline & $\begin{array}{l}\text { Proponer oportunidades de integración a los colectivos en } \\
\text { riesgo de exclusión del mercado de trabajo. }\end{array}$ \\
\hline $\begin{array}{ll}\text { Apoyo } & \text { de } \\
\text { iniciativas } & \\
\text { locales } & \text { de } \\
\text { generación } & \text { de } \\
\text { empleo } & \\
\end{array}$ & $\begin{array}{l}\text { Apoyar las iniciativas locales que contribuyan a la generación } \\
\text { de empleo. }\end{array}$ \\
\hline $\begin{array}{l}\text { Asistencia } \\
\text { técnica }\end{array}$ & $\begin{array}{l}>\text { Gestión puesta en marcha, seguimiento y control. } \\
>\text { Información, equipamiento y evaluación externa. }\end{array}$ \\
\hline
\end{tabular}

\subsection{Iniciativas comunitarias}

Desde la Unión se han puesto en marcha una serie de Iniciativas comunitarias con el objetivo de atender a los colectivos con mayores dificultades de inserción en el mercado de trabajo. Para ello se constituyó la Iniciativa Comunitaria de Empleo y desarrollo de los Recursos Humanos (1994-1999), que cuenta con tres capítulos: NOW, dirigido a mujeres; HORIZON/INTEGRA, destinado a colectivos desfavorecidos y a personas con discapacidad, y YOUTHSTART, dirigido a jóvenes.

- NOW: Se ha dirigido al colectivo de mujeres y su objetivo prioritario ha sido la igualdad de posibilidades y oportunidades para este colectivo, en particular el acceso a actividades en sectores de futuro y posiciones directivas.

- HORIZON/INTEGRA: Se ha dirigido al colectivo de personas con discapacidad, personas desfavorecidas y grupos de riesgo, como personas con problemas de toxicomanías, desempleados de larga duración, minorías étnicas y su objetivo prioritario ha sido la inserción de estos colectivos en el mercado de trabajo.

- YOUTHSTART: Se ha dirigido al colectivo de jóvenes menores de 20 años sin formación profesional básica o cualificación mínima para su incorporación al mercado de trabajo.

Otra iniciativa ha sido $A D A P T$, cuyo objetivo se ha centrado en promover la formación y cualificación de trabajadores en respuesta a las modificaciones del mercado de trabajo.

Las nuevas iniciativas comunitarias aprobadas para el período $2000 / 2006$ son las que se enuncian a continuación, destacando la iniciativa Equal como aquella que incorporará entre sus objetivos los asumidos anteriormente por las iniciativas de empleo, en todos sus capítulos, y la de Adapt:

- INTERREG III: Cooperación transfronteriza, transnacional e interregional, destinada a fomentar un desarrollo armonioso, equilibrado y sostenible del conjunto del espacio comunitario. Se financiará fundamentalmente con los fondos Feder. 
- URBAN: Revitalización económica y social de las ciudades y periferias urbanas en crisis con vistas a promover un desarrollo urbano sostenible.

- EQUAL: Cooperación transnacional para promocionar nuevos métodos de lucha contra las discriminaciones y desigualdades de toda clase en relación con el acceso al mercado de trabajo. Se financiará con el Fondo Social Europeo.

- LEADER: Desarrollo rural mediante programas de desarrollo integrados y de cooperación de los grupos de acción local. Se financiará con los fondos Feoga.

\subsection{El programa comunitario Leonardo}

Como ya se ha enunciado, son muchos los esfuerzos realizados en la Unión Europea por desarrollar una política común de formación profesional. Las estrategias y líneas maestras de esta actuación comunitaria se concretaron durante la segunda mitad de la década de los ochenta en una serie de programas comunitarios:

- Lingua (1990/1994): Programa para el fomento de las competencias idiomáticas en la Comunidad Europea. Tuvo una dotación presupuestaria de 153 millones de ecus.

- Comett I (1986/1989) y Comett II (1990/1994): Programa que tenía por objeto la cooperación entre universidades y empresas para la formación profesional en el campo de las nuevas tecnologías. El primer programa se dotó con 45 millones de ecus y el segundo con 200 millones de ecus.

- Eurotecnet: Fomentar la innovación en el campo de la formación profesional.

- FORCE: Desarrollo de la formación profesional continua en la comunidad europea.

- PETRA: Formación profesional de los jóvenes.

Todos estos programas han pasado a unificarse bajo el programa LEONARDO ${ }^{26}$ que se dirige a garantizar una acción comunitaria en el ámbito de la formación profesional y a facilitar los medios necesarios para afrontar los retos planteados en los años noventa.

El marco común de objetivos, recoge las grandes líneas inspiradoras de la política de formación profesional comunitaria, entre las que cabe destacar la mejora de la calidad y capacidad de innovación de los sistemas; la promoción de la formación a lo largo de toda la vida para responder a las necesidades de los trabajadores de las empresas; el desarrollo y adquisición de competencias clave y lingüísticas; la adaptación de la formación a los cambios sociales y tecnológicos; el uso de las tecnologías de la información dentro de la formación abierta y a distancia; la formación para jóvenes y adultos sin formación y con riesgo de exclusión social y la transparencia de las cualificaciones.

Con el Programa Leonardo se agrupan por primera vez bajo un solo marco, todas las acciones que habían sido objeto de los programas comunitarios (Force, Eurotecnet, Lingua, Petra, Commett, Red Iris) abarcando

26 Propuesta de Decisión del Consejo por el que se establece un programa de acción para la aplicación de una política de formación profesional de la Comunidad Europea LEONARDO da Vinci (Presentada por la Comisión el 4 de febrero de 1994) DO.N. ${ }^{\circ} \mathrm{C} 67$ de 4.3.1994, p. 12. 
con ello todas las dimensiones y ámbitos de la formación profesional:

- Formación Profesional Inicial: Cualquier tipo de formación inicial, incluida la enseñanza técnica y profesional y los sistemas de aprendizaje, que permitan el acceso de los jóvenes a una cualificación profesional reconocida por las autoridades competentes del Estado miembro en el que se haya obtenido.

- Formación Profesional Continua: Cualquier acción de formación profesional realizada por un trabajador de la Unión Europea durante su vida activa.

- Continuum (aprender durante toda la vida): El continuum tiene por objeto la promoción de nuevas formas y dispositivos de formación que fomenten el aprendizaje a lo largo de la vida, lo cual requiere la cooperación transversal entre los diferentes ámbitos y dimensiones de la formación.

- Orientación Profesional: La prestación de consejos e informaciones en lo relativo a la elección y movilidad profesionales.

- Aprendizaje y formación abiertos y a distancia: Cualquier tipo de formación profesional y autónoma que incluya la utilización de tecnologías y servicios de información y de comunicación, de forma tradicional y avanzada y el apoyo de servicios de orientación y tutorías individualizadas.

El Programa se divide en capítulos, cada uno de los cuales desarrolla un determinado bloque de medidas:

- Proyectos piloto transnacionales. Una acción realizada en virtud de una asociación transnacional con objeto de concebir, preparar y experimentar el diseño de métodos, contenidos y materiales de educación y formación profesional; la concepción y puesta en práctica de metodologías de anticipación de necesidades de formación y/o de evaluación de productos y resultados y de métodos e instrumentos de formación profesional; la difusión y transferencia a los sistemas y dispositivos de formación profesional de los métodos, productos, resultados e instrumentos que se deriven de los proyectos piloto transnacionales.

- Programas transnacionales de estancias e intercambios. Acciones transnacionales que mediante la movilidad de las personas participantes tienen como objetivo desarrollar la dimensión europea en la política y en los contenidos de la formación profesional; promover la experiencia profesional práctica; desarrollar las competencias lingüísticas; promover la cooperación entre las diferentes organizaciones y niveles implicados en la formación; facilitar la transferencia de tecnología a la empresas mediante la formación.

- Encuestas y análisis transnacionales e intercambio de datos comparables. serán realizados en el marco de un programa de trabajo aprobado por el Comité Leonardo y estarán orientados a reforzar el desarrollo de los conocimientos en el sector de la formación profesional. 


\section{RETOS Y DESAFÍOS PARA LOS SISTEMAS DE FORMACIÓN PROFESIONAL EUROPEOS}

La política comunitaria de formación profesional se encuentra en una etapa nueva de su construcción. La calidad y los niveles de los sistemas de educación y de formación están en el centro de las preocupaciones en todos los Estados miembros de la Unión Europea.

Las políticas de formación profesional tienen una importancia creciente para dar a los recursos humanos el papel que les corresponda en el mantenimiento del crecimiento económico en Europa. Algunos de los retos y exigencias que se le plantean a los sistemas de formación profesional europeos son los que se expresan a continuación:

- Deben presentarse a un entorno económico y social cada vez más dinámico y menos definido.

- La mundialización y globalización de las empresas ha obligado a reconocer a los recursos humanos como un factor fundamental en las relaciones de competencia que se establecen entre las empresas y los sistemas económicos.

- Buscar mediante las políticas de formación una mayor capacidad y cualificación de sus ciudadanos, garantizando una formación a lo largo de toda la vida.

- Europa necesita realizaciones y aptitudes del más alto nivel en el ámbito del desarrollo y de la utilización de las nuevas tecnologías, por otra parte, sin una suficiente cualificación de la mano de obra, las empresas no pueden desarrollar eficazmente los resultados potenciales de los nuevos procesos de producción y lograr los niveles de competitividad necesarios en los mercados mundiales.

- Una fuerte presión en favor de un desarrollo de las políticas de formación continua, formación dirigida a los trabajadores ocupados, sin reducir el esfuerzo realizado para conseguir una mayor cualidad en la formación de los jóvenes que buscan incorporarse al mercado de trabajo.

- La formación y la cualificación de las mujeres en favor de una igualdad de oportunidades en el acceso a la formación y al empleo cualificado.

- Los déficit de cualificación que mantienen un alto nivel de desempleo y una escasez de mano de obra cualificada.

- Se debe garantizar el acceso a una educación y formación de calidad en todo el entorno europeo, lo que supone la creación de un espacio que facilite la movilidad y la eliminación de fronteras y la transparencia en las cualificaciones profesionales.

\section{ENTIDADES EUROPEAS SIGNIFICATIVAS}

En este apartado se hace referencia a las entidades significativas en el ámbito europeo en cuanto a la formación profesional. La entidad con más peso en este momento es el CEDEFOP, como Centro 
Europeo para el Desarrollo de la Formación profesional en Europa. Existen homólogos en otros continentes, que realizan el mismo trabajo y tienen asignadas prácticamente las mismas funciones, en el entorno en el que actúan. Estos son el CIADFOR ${ }^{27}$ en el ámbito africano y el CINTERFOR, en el ámbito latinoamericano.

También se hace referencia al Comité Consultivo de la Formación profesional, creado cuando se pretendía establecer una política común de formación profesional en Europa. Hoy esa idea no es compartida por todos los Estados miembros, primando el principio de subsidiariedad, que da las competencias en este terreno a los diferentes Estados miembros.

- El CEDEFOP28: Centro Europeo para el Desarrollo de la Formación Profesional, fue creado en 1975. Es una institución descentralizada de la Unión Europea, independiente de la estructura de la Comisión Europea. Su tarea consiste en realizar una asistencia técnica y científica a la Unión Europea para el desarrollo y realización de una política comunitaria de formación profesional. Este Centro recibe sus líneas de trabajo del Consejo de Administración, compuesto por tres miembros de la Comisión Europea y por tres representantes, para cada uno de los quince países, de las organizaciones empresariales, sindicales y de los gobiernos. Las federaciones europeas de los agentes sociales Unión de Industrias de la Comunidad Europea (UNICE) y Confederación Europea de Sindicatos (CES) tienen en el Consejo un representante permanente. Entre sus proyectos se encuentran: descripciones comparativas de los sistemas de cualificación y formación profesional en Europa; punto de encuentro entre la investigación y la práctica; programa europeo de visitas de estudio para especialistas en formación profesional; asistencia a programas europeos de promoción, en particular al programa Leonardo da Vinci.

- Comité Consultivo de la Formación Profesional: fue creado por decisión del Consejo en 1963, en donde establecía los principios generales de una política común de formación profesional, entre los que aparecía la constitución de este Comité integrado por representantes de la administración europea y representantes de las administraciones y de las organizaciones empresariales sindicales de los diferentes Estados miembros. El objetivo de creación es apoyar el desarrollo de esa política común de formación profesional en el entorno europeo.

- Fundación Europea para la Formación Profesional29: comienza su labor en 1994, se sitúa en Turín y su objetivo es apoyar a la Unión Europea en el fomento de la cooperación en formación profesional con los países de la Europa Central y del Este y en la aplicación de los programas Tempus, Tacis y Phare.

\section{REFERENCIAS BIBLIOGRÁFICAS}

CEDEFOP (1996): Glosario: Formación profesional. Berlín, Cedefop.

CEDEFOP (1996): Initial and continuing vocational training in Europe. Thessaloniki, Cedefop.

\footnotetext{
${ }^{27}$ CIADFOR Documentation Centre. PO Box 3771. ABIDJAN 01. Cote d'Ivoire.

28 Reglamento (CEE)N. ${ }^{\circ} 337 / 75$ por el que se crea un Centro Europeo para el desarrollo de la formación profesional. DO.N. ${ }^{\circ} \mathrm{L} 30$ de 9.2.1995, p. 1.

${ }^{29}$ El día 7 de mayo de 1990 el consejo determina la creación de la Fundación Europea de la Formación.
} 
CEDEFOP (1993): Formación para una sociedad en cambio (Informe acerca de la situación actual de la investigación sobre la formación profesional en Europa. Salónica, Cedefop.

Comisión Europea (1997): Chiffres clés sur la formation professionnelle dans l'Union Européenne. Bruselas,

--- (1995): Libro Blanco sobre la educación y la formación: enseñar y aprender. Hacia la sociedad cognitiva. Luxemburgo, Oficina publicaciones oficiales de las Comunidades europeas.

---- (1994): Libro Blanco: crecimiento, competitividad, empleo; retos y pistas para entrar en el siglo XXI. Luxemburgo, Oficina publicaciones oficiales de las comunidades europeas.

---- (1993): La Formación profesional en la Comunidad Europea: desafíos y perspectivas. Luxemburgo, Oficina publicaciones oficiales de las Comunidades europeas.

EURYDICE y CEDEFOP (1995): Estructuras de los sistemas educativos y de formación inicial en la Unión Europea. Luxemburgo, Oficina de Publicaciones oficiales de la Comunidad Europea.

OIT (2000): Informe sobre el empleo en el mundo 1998-1999. Empleabilidad y mundialización papel fundamental de la formación. OIT

UNESCO (2000): Informe sobre la educación en el mundo. El derecho a la educación, hacia una educación para todos a lo largo de la vida. Madrid, Grupo Santillana/Ediciones UNESCO.

\section{Legislación europea}

Dêcision du Conseil du 2 avril 1963 portant établissement des principes généraux pour la mise en oeuvre d'une politique commune de formation professionnelle. JO. 63 du 20.4.1963, p. 1338/63.

Règlement (CEE) n 337/75 du conseil du 10 février 1975. portant crétion d'un centre européen pour le dévoloppment de la formation professionelle. JO. L 39. du 13.2.1975, p. 1.

Recomendación de la Comisión, de 6 de julio de 1977, a los Estados miembros relativa a la preparación profesional de los jóvenes en paro o amenazados de perder su empleo. DO L 180 de 20.07.1977

Résolution du Conseil du 18 décembre 1979 concernant la formation en alternance des jeunes. JO. C 1. du 3.1 .80$, p.1.

Décision du conseil du 16 juillet 1985 concernant la correspondance des qualifications de formation professionnelle entre Etats membres des Communautés européennes. JO. L 199. du 31.7.85, p. 56.

Résolution du conseil et des ministres de l'education, réunis au sein du conseil du 5 décembre 1985 prorogeant d'une année certaines mesures prises en vue d'améliorer la preparation des jeunes à l'activite professionnelle et de faciliter leur passage de l'educatión à la vie active. JO. C 320. du 18.12.85, p. 3.

Conclusiones del Consejo de 9 de marzo de 1987 relativas a la formación profesional de jóvenes en la Comunidad Europea. DO. C 73 de 20.3.1987, p. 2.

Decisión del Consejo de 1 de diciembre de 1987 sobre un programa de acción para la formación y la preparación de los jóvenes para la vida adulta y profesional. DO. L 346 de 10. 12.1987, p. 31.

Conclusiones del Consejo y de los ministros de educación reunidos en el Consejo sobre el segundo programa de acción de la Comunidad Europea (1982-1987) sobre la transición de los jóvenes de la enseñanza a la vida adulta y activa de 24 de mayo de 1988. DO. C 177 de 6. 7.1988, p. 1.

Resolución del Consejo de 5 de junio de 1989 sobre la formación profesional permanente. DO. C 148 de 15. 6.1989, p. 1.

Conclusiones del Consejo y de los ministros de educación reunidos en Consejo de 14 de diciembre de 1989 sobre la enseñanza y la formación inicial en el ámbito técnico y profesional. DO. C 27 de 6. 2.1990, p. 4. 
Resolución del Consejo de 18 de diciembre de 1990 sobre la correspondencia de las cualificaciones de formación profesional. DO. C 109 de 24.04.1991, p.1.

Directiva 92/51/CEE del Consejo de 18 de junio de 1992 relativa a un segundo sistema general de reconocimiento de formaciones profesionales, que completa la Directiva 89/48/CEE. DO. L 209 de 24. 7.1992, p. 25.

Resolución del Consejo de 3 de diciembre de 1992 sobre la transparencia de las cualificaciones profesionales. DO. C 49 de 19. 2.1993, p. 1.

Dictamen sobre la propuesta de Recomendación del Consejo sobre el acceso a la formación profesional continua. DO. C 129 de 10. 5.1993, p. 57.

Resolución del Consejo de 11 de junio de 1993 relativa a la formación profesional para los años noventa. DO. C 186 de 8.7.1993, p. 3.

Recomendación del Consejo de 30 de junio de 1993 sobre el acceso de la formación profesional permanente. DO. L 181 de 23.7.1993, p. 37.

Dictamen sobre el proyecto de decisión por la que se establece un Programa de acción para la aplicación de una política de formación profesional de la Comunidad Europea. DO. C 148 de 30.5.1994, p. 5.

Decisión del Consejo de 6 de diciembre de 1994 por la que se establece un programa de acción para la aplicación de una política de formación profesional de la Comunidad Europea. DO. L 340 de 29.12.1994, p. 8.

Resolución del Consejo de 5 de diciembre de 1994 sobre la calidad y atractivo de la formación profesional. DO. C 374 de 30.12.1994, p. 1.

Propuesta modificada de Decisión del Parlamento Europeo y del Consejo por la que se establece el Año Europeo de la Educación y la formación a lo largo de toda la vida (1996). (Presentada por la Comisión con arreglo al apartado 2 del artículo 189 A del Tratado CE el 31 de marzo de 1995. DO. C 134 de 1.6.1995, p. 6.

Conclusiones del Consejo de 24 de julio de 1995 relativas a la importancia y a los retos de la formación profesional. DO. C 207 de 12.8.1995, p. 7.

Conclusiones del Consejo de 20 de diciembre de 1996 sobre una política de educación permanente. DO. C 7 de 10.1.1997, p. 6.

Consejo Europeo de Luxemburgo. «Tratado de Amsterdam». Luxemburgo 20.10.1997

Decisión del Consejo, de 26 de abril de 1999, por la que se establece la segunda fase del programa de acción comunitario en materia de formación profesional Leonardo da Vinci (99/382/CE). DO L 146 de 11.06.1999 